\title{
FILOSOFÍA DEL DERECHO NATURALIZADA: POSIBILIDAD Y REFUTACIÓN*
}

\author{
PHILOSOPHY OF NATURALIZED LAW: \\ POSSIBILITY AND REFUTATION
}

\section{Camilo Humberto Prieto Fetiva**}

\section{Resumen}

Uno de los problemas actuales de la teoría del derecho gira en torno a la metodología que debe usar, en tal sentido las discusiones se centran en dos extremos: i) posturas que buscan mantener el método clásico del análisis conceptual, ii) posiciones que persiguen una interdisciplinariedad, lo que trae consigo la incorporación de nuevos métodos. Dentro del punto ii) se sitúa la denominada filosofía del derecho naturalizada propuesta por Leiter, la cual a su vez se basa en el realismo jurídico norteamericano y en el giro naturalista de Quine e igualmente, tiene como finalidad elevar los estándares de validación empírica de las tesis ius-filosóficas.

Así las cosas, el objetivo principal de este trabajo es el de lograr una clarificación conceptual respecto a la propuesta de una filosofía del derecho naturalizada de Brian Leiter así como analizar las posibles ventajas y problemas que se desencadenan con su aplicación en el campo de la teoría del derecho. En tal sentido, este artículo se dividirá en tres partes: i) el giro naturalista en la investigación filosófica y planteamientos genéricos del realis-

Artículo recibido el 24 de junio de 2019 y aceptado para su publicación el 10 de julio de 2020. Ganador del IV Concurso de Investigación en Filosofía del Derecho de Estudiantes de Posgrado: paradigmas emergentes en derecho y filosofía jurídica.

El presente artículo hace parte del trabajo de investigación realizado por el autor en el grupo de estudios legales y sociales "Phronesis", adscrito al centro de investigaciones socio jurídicas de la Universidad Católica de Colombia (CISJUC), en el marco del proyecto de investigación titulado: Derechos Humanos, Interculturalidad y Ética Pública. Fase 2.

** Docente de hora cátedra de la Facultad de Derecho de la Universidad Católica de Colombia ORCID https://orcid.org/0000-0002-5040-3444, Correo electrónico: camilohumberto@hotmail.com.chprieto@ucatolica.edu.co. 


\section{CAMILO HUMBERTO PRIETO FETIVA}

mo jurídico norteamericano, 2) el neo realismo jurídico de Brian Leiter y la naturalización de la teoría jurídica, 3) problemas y críticas de la filosofía del derecho naturalizada.

\section{Palabras clave:}

Naturalización de la filosofía del derecho, predictibilidad de las decisiones judiciales, realismo jurídico, naturalismo, validación empírica.

\section{$\underline{\text { Abstract }}$}

One of current problems in legal theory revolves around the methodology to be used. As such, discussions focus on two extremes: i) positions that seek to preserve the traditional method of conceptual analysis, and ii) positions that pursue an interdisciplinary approach, which entails the incorporation of new methods. Point ii) encompasses the so-called naturalized legal philosophy proposed by Leiter, which is in turn based on US legal realism and Quine's naturalistic perspective while also aiming to raise the standards of empirical validation of jus-philosophical theories.

The main objective of this paper is to clarify conceptual aspects of Brian Leiter's proposal of a naturalized legal philosophy, as well as to analyze the possible advantages and problems that may arise from its application in the field of legal theory. This paper is divided into three parts: 1) the naturalistic shift in philosophical research and generic approaches of US legal realism, 2) Brian Leiter's new legal realism and the naturalization of legal theory, and 3) problems and criticism of naturalized legal philosophy.

\section{Keywords:}

Naturalization of the Philosophy of Law, Predictability of Judicial Decisions, Legal Realism, Naturalism and Empirical Validation. 
FILOSOFÍA DEL DERECHO NATURALIZADA...

SUMARIO: I. Introducción. II. El giro naturalista en la investigación filosófica y planteamientos genéricos del realismo jurídico norteamericano. III. El neo-realismo jurídico de Brian Leiter y la naturalización de la teoría jurídica. IV. Problemas y críticas de la filosofía del derecho naturalizada. V. Conclusiones. VI. Referencias.

\section{INTRODUCCIÓN}

Cuando se habla de filosofía del derecho naturalizada por lo general las personas que no han tenido un acercamiento a la misma suelen relacionarla con el derecho natural, el cual tiene como fundamento el hecho, de que el derecho puede derivarse, interpretarse y aplicarse a partir de la idea de naturaleza humana. ${ }^{1}$ No obstante, no existe una interpretación unívoca acerca de la idea de naturaleza humana, lo que ha marcado diferentes acepciones del derecho natural y su misma evolución hasta la fecha ${ }^{2}$ donde se erigen como representantes principales de su versión contemporánea Carlos Massini, ${ }^{3}$ y John Finnis. ${ }^{4}$

Por el contrario, la filosofía del derecho naturalizada propuesta por Brian Leiter, ${ }^{5}$ tiene una concepción complementaria al positivo

1 Welsel, Hans, Introducción a la filosofía del derecho: derecho natural y justicia material, 2a. ed., trad. de Felipe González Vincén, Madrid, Aguilar, 1979.

2 Carpintero, Francisco, Historia del derecho natural: un ensayo, México, UNAM, 1999.

3 Massini, Carlos, La Ley natural y su interpretación contemporánea, Pamplona, EUNSA, 2006.

4 Finnis, John, Estudios de teoría del derecho natural, edición de Javier Saldaña y Carlos Massini, México, UNAM, 2017.

5 La postura que se acogerá de naturalización filosófica es la de Brian Leiter, la cual a su vez se fundamenta en el giro naturalista de Quine, sin embargo, existen otras visiones de naturalización tanto jurídicas como filosóficas, al respecto véase: Kuhn, Thomas, La estructura de las revoluciones científicas, traducción de Agustín Contin, Buenos Aires, Fondo de Cultura Económica, 2004; Maddy, Penelope. Second Philosophy: A Naturalistic Method, Oxford, Oxford University Press, 2007; González, Daniel, "Filosofía y (Neuro)ciencia: Sobre la "naturalización" de la filosofía práctica”, ponencia presentada en el primer congreso de filosofía del dere- 


\section{CAMILO HUMBERTO PRIETO FETIVA}

jurídico, debido a que uno de sus objetivos principales es una idónea representación del proceso de decisión judicial, ${ }^{6}$ e igualmente se enmarca en el denominado positivismo jurídico excluyente, ${ }^{7}$ lo que hace que ésta, no sea equivalente a los postulados iusnaturalistas. Ésta forma de entender la filosofía jurídica, tiene sus fundamentos en el denominado giro naturalista de la filosofía y en el realismo jurídico norteamericano, pese a ello no es una reiteración histórica de los planteamientos de dicho movimiento sino, por el contrario, es fruto de una reconstrucción y desarrollo de los postulados de este, lo que hace que tenga una connotación de neo-realismo ${ }^{8}$ - el cual no debe confundirse con el denominado New Legal Realism- ${ }^{9}$

A su vez se instituye como una disciplina que va más allá del análisis conceptual predominante en la filosofía del derecho tradicional, entendido este como el análisis que se realiza acerca de la organización y de los componentes del derecho a saber, las normas y los conceptos jurídicos ${ }^{10}$. Para ello, se apoya en la aplicación en la teoría jurídica de los métodos de validación empírica propios de las ciencias

cho para el mundo latino, Alicante, 2016, pp. 1-31. http://iusfilosofiamundolatino. ua.es/download/Filosof\%C3\%ADa\%20y\%20(Neuro)ciencia.\%20Sobre\%20la\%20 naturalizaci\%C3\%B3n\%20de\%20la\%20filosof\%C3\%ADa\%20pr\%C3\%A1ctica.pdf.

6 Ratti, Giovanni, "Estudio introductorio", en Leiter, Brian, Naturalismo y teoría del derecho, Madrid, Marcial Pons, 2012, pp. 11-27.

7 Leiter, Brian, “Objectivity, Morality, and Adjudication", en Leiter, Brian, Objectivity in law and morals, Cambridge, Cambridge University Press, 2007, pp. 66-98.

8 Núñez, Álvaro, "El realismo jurídico de Brian Leiter", Diritto e questioni pubbliche, Palermo, 2010, núm. 10, pp. 438-456.

9 El denominado New Legal Realismo, es una escuela jurídica norteamericana que al igual que la postura esbozada por Leiter es heredera del realismo jurídico norteamericano, esta se caracteriza por prestar un especial interés al papel que juega el derecho en la cotidianidad de las personas, esto es, el rol que tiene el derecho en las sociedades contemporáneas, en tal sentido, para realizar tal actividad esta visión enfatiza en la interdisciplinariedad del derecho, así como la armonización entre los métodos cuantitativos y cualitativos de investigación. Mertz, Elizabeth, "New Legal Realism: Law and Social Science in the New Millennium", en Mertz, Elizabeth et al., The New Legal Realism, Volume I. Translating law-and-society for today's legal practice, Cambridge: Cambridge University Press, 2016, pp. 1-25.

10 Bix, Brian, "Algunas reflexiones sobre metodología en Teoría del Derecho" traducción de Pablo Navarro, Doxa. Cuadernos de filosofía del derecho, Alicante, 2003, núm. 26, pp. 609-633. 
FILOSOFÍA DEL DERECHO NATURALIZADA...

naturales y sociales ${ }^{11}$ lo que trae consigo un aumento en el estándar de validación de las teorías, ${ }^{12}$ situación que supone un avance respecto al análisis conceptual.

Sin embargo, lo anterior no es del todo claro ya que no se sabe con exactitud en qué momento se ésta ante la presencia de una filosofía del derecho naturalizada, es decir ¿Cuál es la distinción característica de ésta respecto al realismo jurídico? ¿En qué consiste su aplicación en el campo jurídico? ¿Cuál es su avance en términos de metodología de la investigación jurídica respecto al análisis conceptual? ¿Qué retos enfrenta?

En este sentido, el objetivo principal de este trabajo es lograr una clarificación conceptual respecto a la propuesta de una filosofía del derecho naturalizada de Brian Leiter y analizar las posibles ventajas y problemas que se desencadenan con su aplicación en el campo de la teoría del derecho. Para ello se abordaran los siguientes tópicos: en primer lugar se analizaran los fundamentos de la filosofía del derecho naturalizada: el giro naturalista en la investigación en la investigación filosófica y los planteamientos genéricos del realismo jurídico norteamericano, en segundo lugar, se abordara de manera critica el neo-realismo de Brian Leiter y su propuesta de naturalización de la teoría jurídica, para luego, en tercer lugar, considerar los principales problemas y retos que enfrentan tales planteamientos y finalmente se expondrán las conclusiones que arrojo el presente trabajo.

No sobra decir que el planteamiento de Leiter es propio de una tradición de derecho anglosajón, que, a su vez, tiene ciertos elementos en común con la teoría jurídica analítica de la escuela genovesa, la cual es, propia de una tradición jurídica de derecho continental, lo que implica una conversación teórica entre dos posturas teóricas similares que responden a una fundamentación diferente. ${ }^{13} \mathrm{E}$ igual-

11 Leiter, Brian, "Rethinking Legal Realism: toward a Naturalized Jurisprudence”, Texas Law Review, Austin, vol. 76, num. 2, diciembre de 1997, pp. 267-315.

12 Cáceres, Enrique y Montemayor Carlos, "Pasos hacia una naturalización cognitiva en la filosofía del derecho (indexicalidad consciente e inconsciente: una revisión a la eficacia normativa)", Problema. Anuario de filosofía y teoría del derecho, México, núm. 10, enero-diciembre de 2016, pp. 137-165.

13 Mientras la obra de Leiter recoge las posturas del realismo jurídico nortea- 
mente, vale pena aclarar que pese a las distinciones existentes entre teoría jurídica y filosofía del derecho, ${ }^{14}$ de las que se derivan el punto de vista interno - teoría jurídica- y externo - filosofía del derecho-, el presente trabajo acoge una visión Ferrajoliana, mediante la cual la teoría jurídica actual involucra juicios de validez — punto de vista interno-, como juicios de legitimidad — punto de vista externo-. ${ }^{15}$

\section{EL GIRO NATURALISTA EN LA INVESTIGACIÓN FILOSÓFICA Y PLANTEAMIENTOS GENÉRICOS DEL REALISMO JURÍDICO NORTEAMERICANO}

No es posible hablar de una filosofía del derecho naturalizada sin abordar las dos principales corrientes que la fundamentan, en primer lugar, se halla el giro naturalista de la filosofía, el cual nace como antítesis al "giro lingüístico", el cual propende porque los problemas filosóficos tradiciones sean abordados desde el lenguaje, lo que acarrea que los filósofos que se acogen a esta forma de entender la filosofía analicen los conceptos propios de la misma. ${ }^{16}$ Bajo esta visión los problemas filosóficos son problemas del lenguaje.

Debido a esta situación Willard Van Orman Quine formula el "giro naturalista” de la filosofía, que consiste en que las preguntas filosóficas deben ser reemplazadas por preguntas científicas de carácter

mericano, la escuela Genovesa hace un estudio tanto del realismo jurídico norteamericano como del escandinavo y a partir de éstos y de la obra de Giovanni Tarello, genera una nueva forma de entender el realismo jurídico, denominada como el realismo jurídico genovés, al respecto véase Barberis, Mauro, "Un poco del realismo jurídico Genovés", en Ferrer, Jordi y Ratti, Giovanni (ed.), El realismo jurídico genovés, Madrid, Marcial Pons, 2011, pp. 201-216.

${ }_{14}$ Guastini, Riccardo, Distinguiendo: estudios de teoría y metateoría del derecho, traducción de Jordi Ferrer Beltrán, Barcelona, Gedisa, 1999.

15 Ferrajoli, Luigi, Principia iuris. Teoría del derecho y de la democracia, 2a. ed., trad. de Perfecto Andrés Ibáñez, Alfonso Ruiz Miguel et al., Madrid, Trotta, 2016, t 1. Teoría del derecho.

16 Leiter, Brian, "The naturalistic turn in legal philosophy", Apa newsletter on philosophy and law, Newark, 2001, vol. 00, num. 2, Spring. 
empírico, ${ }^{17}$ dicho planteamiento trae consigo una yuxtaposición de la ciencia sobre la filosofía, debido a que, el obtener conocimiento ya no reside en la delimitación-limitación que se realice sobre la forma de ver el mundo, si no que ahora implica la existencia de una ciencia filosófica, ${ }^{18}$ la que busca que el saber filosófico sea parte de la ciencia, y no una disciplina apartada de la misma. ${ }^{19}$

En este sentido, lo anterior enlaza los siguientes postulados: i) La idea de naturalismo provoca que la imagen científica acerca del origen y existencia del ser humano adquiera una fiabilidad elevada sobre sus sistemas cognitivos, ${ }^{20}$ ii) las preguntas acerca de la naturaleza del conocimiento humano deben incluir consideraciones respecto a las capacidades psicológicas del mismo y a su evolución, dado que, si este presupuesto no se cumple, se genera poca plausibilidad a dichos planteamientos. ${ }^{21}$

Tal situación se debe a que Quine, entiende a la epistemología como a aquella disciplina que se ocupa de la fundamentación de la ciencia, que puede ser realizada de dos maneras, la primera es por medio de los estudios conceptuales, y la segunda es a través de los estudios doctrinales. ${ }^{22}$ Los estudios conceptuales se ocupan de la clarificación de los conceptos empleados en la fundamentación, mientras que los estudios doctrinales, estriban sobre la validación empírica de las leyes que se formulan. ${ }^{23}$ Así pues, la propuesta epistemológica de

17 Quine, W., "Naturalización de la epistemología”, trad. de Manuel Garrido, en Quine, W., La relatividad ontológica y otros ensayos, Madrid, Tecnos, 2002, pp. 93119.

18 López, Nicolás, "Naturalismo y derecho: una perspectiva científica en la teoría del derecho norteamericano del siglo XXI", Revista de Ciencias Sociales, Valparaíso, 2016, núm. 68, pp. 13-36.

19 Magge, Bryan, “Las ideas de Quine. Diálogo con W. V. Quine”, en Magge, Bryan (ed.), Los hombres detrás de las ideas. Algunos creadores de la filosofía contemporánea, Mexico, Fondo de Cultura Económica, 1993, pp. 177-189.

20 López, Nicolás, Naturalismo y derecho..., cit., p, 20.

21 Ídem.

22 Quine, W, op. cit., p, 93.

23 Ibidem, p. 93.94. 


\section{CAMILO HUMBERTO PRIETO FETIVA}

Quine tiene una correlación con la ciencia natural, en la medida en que va unida tanto a la evidencia como a la verificación. ${ }^{24}$

Por esta razón, se está ante la presencia de la naturalización de la filosofía si y solo si la tarea central de la ciencia como de la filosofía es abordada como un problema empírico que debe abordarse de forma científica. ${ }^{25}$ En palabras de Bertrand Russell:

El verdadero método, tanto en la filosofía como en la ciencia, será inductivo, minucioso, respetuoso del detalle sin creer que cada filósofo debe resolver los problemas por sí mismo. Es este método que inspira el realismo analítico, y es solo mediante el, si no me equivoco, que la filosofía logrará éxito en obtener resultados tan solidos como los de la ciencia. ${ }^{26}$

En segundo lugar, se encuentra el realismo jurídico estadounidense que nace aproximadamente en 1918 y logra su consolidación en la década de los $20 .{ }^{27}$ Éste tiene sus orígenes en los trabajos escépticos sobre el proceso judicial que publicaron John Chipman Gray $^{28}$ y posteriormente Oliver Wendell Holmes, ${ }^{29}$ en los cuales se refutó al formalismo jurídico, ${ }^{30}$ predominante en la época gracias a Christopher Langdell, lo que marca una distinción con los otros realismos, puesto que el escandinavo nace como contraposición al

24 Ibidem, pp. 100-118,

25 López, Nicolás, "El naturalismo jurídico como teoría del derecho: red filosófica y conceptual de un cientificismo revisitado", Derecho y humanidades, Santiago, núm. 22, julio-diciembre de 2013, p. 203.

26 Russell, Bertrand, Análisis filosófico, trad. de Francisco Rodríguez Consuegra, Barcelona, Paidós, 1999, p. 74.

27 Zaremby, Justin, Legal Realism and American Law, Nueva York, Bloomsbury, 2014.

28 Gray, J, The Nature and Sources of the Law, Nueva York, The Columbia University press, 1909.

29 Holmes, Oliver, La senda del derecho, trad. de José Ignacio Solar Cayón, Madrid, Marcial Pons, 2012, p. 55.

30 Los realistas jurídicos norteamericanos - y los realistas en general- también estaban en contravía con el derecho natural, puesto que éstos parten de un concepto de derecho positivo, al respecto véase, Núñez, Álvaro, “Ciencia jurídica realista: modelos y justificación", Doxa. Cuadernos de filosofía del derecho, Alicante, 2012, núm. 35, p. 725. 
FILOSOFÍA DEL DERECHO NATURALIZADA...

idealismo borstromiano y el genovés como oposición al positivismo formalista y al neo-constitucionalismo. ${ }^{31}$

Langdell es considerado el creador de la ciencia jurídica en Estados Unidos y fue el primer decano de la facultad de derecho de la Universidad de Harvard. Éste, formuló tres cualidades que deben tener los sistemas jurídicos: 1) Formalidad y determinación del derecho, de tal modo que las decisiones jurídicas se deriven de forma mecánica por medio del silogismo judicial, y no a partir de cuestiones valorativas, 2) coherencia del sistema jurídico, esto es, que en el mismo no existan vacíos legales - lagunas normativas-, ni contradicciones entre normas - antinomias - y 3) autonomía del sistema jurídico en cuanto al sistema de fuentes, es decir las fuentes aplicables para resolver un caso son las que proporcione el derecho y no otras disciplinas como la filosofía, la moral o la economía política. ${ }^{32}$

Además del anterior aporte, Langdell también contribuyó a la formulación del case method, cuya aplicación robusteció la idea formalista en los Estados Unidos. Este método consistía en lo siguiente: Primeramente, existía una serie de casos que la ciencia legal construía, y se agrupaban de acuerdo con ciertas materias, por ejemplo: derecho de seguros, derecho de sucesiones, derecho de los contratos entre otros. En su segundo momento, estos casos eran expuestos por el docente, o se les pedía a los estudiantes que los leyeran, no de forma completa — sin la solución propuesta por la ciencia legalsino principalmente los hechos y algunas consideraciones preliminares. En un tercer momento, se genera un debate bajo el método socrático, mediante el cual se procura que los estudiantes piensen como abogados y le den una solución al mismo. Y, por último, el docente comparte la solución del caso con su grupo de estudiantes. ${ }^{33}$ Este método, es asimilable al modo de pensar sistemático ${ }^{34}$ de los

31 Idem.

32 López, José, "El formalismo en la teoría jurídica estadounidense", Anuario de filosofía del derecho, Valencia, 2001, núm. XVIII, pp. 267-300.

33 Lavilla, Juan, "Sobre el "case-method" para la enseñanza del Derecho. La experiencia de la "Harvard Law School"”, Revista de Administración Pública, Madrid, 1988, núm. 117, Septiembre-Diciembre de 1988, pp. 433-446.

34 El modo de pensar sistemático según enuncia Viehweg citando a Hartmann, "procede desde el todo, la concepción es en él lo principal y permanece siempre 
códigos propios de la tradición jurídica de derecho continental, dado que solo incluye casos jurídicamente relevantes que no acarreen reflexiones de carácter filosófico ni moral, puesto que, para Langdell esto compete a otras facultades. ${ }^{35}$ Así las cosas, en vez de memorizar y analizar códigos - propio del modelo de enseñanza del derecho positivo - ${ }^{36}$ se memorizan y analizan casos.

En contraposición al formalismo, los realistas, cuyas principales figuras fueron Karl Nickerson Llewellyn, Underhill More, Jerome Frank, Walter Wheeler Cook, Herman Oliphant y León Green, ${ }^{37}$ rompen con la imagen idealizada del juez, ${ }^{38}$ es decir un juez sin preferencias, ni sentimientos, en vista de que estiman que el decisor al momento de resolver un caso determinado no lo hace con fundamento a normas jurídicas sino que, lo hace mediante sus gustos políticos y morales, y después racionaliza su decisión. Lo que acarrea que las valoraciones tanto políticas como morales tengan relevancia en el proceso de decisión judicial. De ahí, que se le atribuya al realismo jurídico estadounidense aquella célebre frase por medio de la cual, "la justicia depende de lo que el juez desayunó". 39

como lo dominante. No hay que buscar un punto de vista. El punto de vista está desde el principio adoptado, y desde él se seleccionan los problemas. El contenido del problema, que no se concilia con el punto de vista, se rechaza y se considera como una cuestión falsamente planteada. Hay que decidir con carácter previo algo que se refiera no a la solución del problema, sino a los limites dentro de los cuales esta solución pueda moverse". Viehweg, Theodor, Tópica y jurisprudencia, 2a. ed., trad. de Luis Díez-Picazo, Madrid, Editorial Aranzadi, 2007, p. 59.

35 Timm, Ana, "Antiformalismo jurídico, aproximaciones básicas", Revista de Derechos Fundamentales, Viña del Mar, 2014, núm. 11, pp. 195-226.

${ }_{36}$ Agudelo, Oscar et al., Teoría jurídica y enseñanza del derecho, Bogotá, Universidad Católica de Colombia, 2015, p. 7.

37 Leiter, Brian, "Realismo jurídico estadounidense", trad. de Jorge Fabra, en Fabra, Jorge y Núñez, Álvaro (ed.), Enciclopedia de filosofía y teoría del derecho, México, UNAM, 2015, vol. 1, pp. 241-276.

38 Ezquiaga, Francisco, "Los juicios de valor en la decisión judicial", Anuario de filosofía del derecho, Valencia, 1984, núm. I, pp. 33-59.

39 No se sabe con certeza cual es el origen de esta frase, sin embargo, algunos historiadores suelen atribuírsela a Jerome Frank, dado que este a diferencia de otros realistas, parte de un escepticismo ante las normas jurídicas, en la media en que estas a su juicio, no son relevantes en el proceso de toma de decisión y se centra en como la personalidad del juez y los hechos del caso, los cuales determinan en es- 
Paralelamente, demandaban un enfoque científico que permitiera establecer lo que los operadores judiciales hacen, más no lo que dicen, y también ayudara a determinar el impacto de sus decisiones sobre la comunidad, ${ }^{40}$ puesto que su objetivo principal es la predicción de decisiones judiciales, al respecto téngase en cuenta lo enunciado por Oliver Wendell Holmes: "El objeto de nuestro estudio, entonces, es la predicción: la predicción de la incidencia de la fuerza pública a través de la actuación de los tribunales". ${ }^{41}$ En tal sentido, el realismo jurídico es una teoría plausible en la medida en que cumple con los dos requisitos señalados por Stephen Hawking ${ }^{42}$ 1) describir el fenómeno que se observa, en este caso la forma en la que fallan los jueces y 2) hacer predicciones sobre el fenómeno observado, es decir, predecir las decisiones judiciales que los operadores proferirán en el futuro.

Otra de sus tesis principales fue la de enunciar que el derecho no es un sistema ni determinado, ni omnicomprensivo, lo que implica que no toda acción esté preestablecida en el ordenamiento jurídico ${ }^{43}$, lo que a su vez genera lagunas ${ }^{44} \mathrm{o}$ antinomias en el mismo, debido a que el derecho es un sistema indeterminado. Situación que reafirma la presencia de juicios de valor en la decisión judicial. Igualmente, las tesis realistas se dieron a conocer por diversas razones:

tricto la decisión tomada por el operador jurídico. Al respecto véase Frank, Jerome, Law \& the modern mind, New Brunswick, Transaction Publishers, 2009.

40 Tumonis, Vitalius, "Legal realism \& judicial decision-making", Jurisprudencija, Wrocławski, 2012, vol. 19, núm. 4, pp. 1361-1382.

41 Holmes, Oliver, op. cit., p, 55.

42 Hawking, Stephen y Mlodinow, Leonard, Brevísima historia del tiempo, trad. de David Jou, Barcelona, Crítica, 2015, p. 208.

43 Redondo, María, "Teorías del derecho e indeterminación normativa", Doxa. Cuadernos de filosofía del derecho, Alicante, 1997, núm. 20, pp. 177-196.

44 Las cuales pueden ser normativas, axiológicas, de conocimiento, de reconocimiento y técnicas, las cuatro primeras son propuestas por Alchourrón y Bulygin y la Quinta es aportada por Guastini, al respecto véase, Alchourrón, Carlos y Bulygin, Eugenio, Sistemas normativos. Introducción a la metodología de las ciencias jurídicas, 2a. ed., Buenos Aires, Editorial Astrea, 2012. Guastini, Riccardo, La sintaxis del derecho, trad. de Álvaro Núñez, Madrid, Marcial Pons, 2016. 
i) Debido a que la mayoría de sus representantes fueron académicos, no obstante, existen algunas excepciones como es el caso de Jerome Frank quien se desempeñó como abogado litigante y posteriormente funcionario público en la administración de Franklin Delano Roosevelt, e igualmente llegó a ocupar el cargo de juez federal. ${ }^{45}$

ii) A debates que sostuvieron con las diversas figuras de la teoría jurídica anglosajona del siglo XX como lo son Herbert Hart, ${ }^{46}$ Ronald Dworkin, ${ }^{47}$ y algunos miembros de los estudios críticos del derecho, lo que generó una distorsión de sus postulados, situación que será analizada en el siguiente acápite.

iii) Al fuerte impacto que ocasionó en los modelos de pedagogía jurídica, lo que a su vez también irradió las reformas judiciales en los Estados Unidos y el ejercicio mismo de la abogacía. ${ }^{48}$

\section{EL NEO REALISMO JURÍDICO DE BRIAN LEITER Y LA NATURALIZACIÓN DE LA TEORÍA JURÍDICA}

Brian Leiter toma como presupuesto fundamental para la construcción de su propuesta las corrientes analizadas anteriormente, en primer lugar en cuanto al realismo jurídico se refiere, Leiter estima que la imagen que genera el realismo partir de diversos teóricos que no hacen parte del movimiento como lo son: Ronald Dworkin, John Hart Ely, Steven Burton, Frederich Shaver, Robert Satter entre otros - que ya fue analizada en este escrito-, es que ésta corriente principalmente se caracteriza por: Formular una teoría descriptiva acerca del proceso de decisión judicial, en el que los operadores judiciales son discrecionales, debido a que toman su decisión con base

45 Leiter, Brian, Realismo jurídico estadounidense, cit., p, 243.

46 Sobre el particular téngase en cuenta: Hart, Herbert, El concepto de derecho, 3a. ed., trad. de Genaro Carríó, Buenos Aires, Abeledo Perrot, 2009, p. 155-193.

47 Al respecto véase: Dworkin, Ronald, Los derechos en serio, trad. de Marta Gustavino, Barcelona, Ariel, 2014, p. 43-60.

48 Leiter, Brian, Realismo jurídico estadounidense, cit., p. 241. 
a sus preferencias personales y luego racionalizan tal decisión con base al ordenamiento jurídico. ${ }^{49}$

Lo anterior se debe a que la imagen que genera el realismo constituye su núcleo central también denominado la tesis nuclear, sin embargo, es un error reducir el realismo a su núcleo, puesto que éste es mucho más que eso. Tal situación se debe a la Franksificación del realismo jurídico norteamericano, es decir, considerar que este movimiento solo se reduce a la figura y a los planteamientos de Jerome Frank sin tomar en cuenta los aportes de otros realistas como Llewellyn o Moore. ${ }^{50}$ La llamada Franksificación parte de la siguiente formula: "Estímulos que afectan al juez x personalidad del juez = decisión",51 la que a su vez implica una ruptura con la fórmula propia del formalismo jurídico: "Normas $\mathrm{x}$ hechos = Decisión". ${ }^{52}$ Por ejemplo:

Fórmula de Frank:

Se presenta en el juzgado $\mathrm{H}$ un caso de custodia del menor A entre la señora L y el señor C.

Estímulos que afectan al juez: La jueza K se levantó malhumorada debido a una discusión que tuvo con su padre respecto a sus tendencias filosóficas.

Personalidad del juez: K se considera feminista, y además estima que la custodia de un menor cuyos padres están divorciados siempre debe de estar en cabeza de la madre.

Decisión: $K$ decide otorgarle la custodia la señora $L$ respecto del menor A.

Fórmula tradicional:

- Norma: En el caso de padres separados la custodia de los hijos menores de edad debe estar en cabeza del padre que este en mejor posición económica para la manutención de estos.

49 Leiter, Brian, Rethinking legal realism..., cit., p, 268.

50 Ibidem, p, 269.

51 Frank, Jerome, "Are judges human? Part two: as through a class darkly", University of Pennsylvania Law Review, Philadelphia, vol. 80, núm. 2, diciembre de 1931, p. 242.

52 Idem. 
- Hechos: Se presenta en el juzgado H un caso de custodia del menor A entre la señora $L$ y el señor $C$.

-Decisión: Teniendo en cuenta el material probatorio obrante en el caso, se determina que la señora L está en una mejor posición económica, por lo que se le otorga la custodia de su hijo A.

Como consecuencia de lo anterior - y más aún cuando las dos fórmulas llevan a una misma decisión - se genera el debate entre realistas y formalistas, que radica en el hecho de establecer qué tipo de razones determinan la decisión judicial, ${ }^{53}$ es decir, si son razones de carácter jurídico, como lo son las normas jurídicas aplicables al caso y los hechos que dan lugar al mismo o si por el contrario, son de tipo extra-jurídico, o extra legal como es el caso de las valoraciones personales del operador judicial, entre otras.

No obstante, pese a esto, Leiter considera que, a partir de planteamientos propios del realismo jurídico, diferentes a los de Jerome Frank, es posible formular una teoría neo-realista del derecho que destaca una teoría descriptiva acerca de la naturaleza de la decisión judicial. Dicha posición no es simplemente una reiteración de planteamientos realistas, sino más bien una reconstrucción teórica de estos, de ahí que tenga el carácter de neo-realismo. Ésta se basa en tres postulados: i) las decisiones judiciales dependen de determinados patrones sociológicos, ii) estos patrones hacen que los jueces sometan su decisión con base a los hechos del caso, iii) se da una racionalización la cual puede ser posterior o en el momento, de la decisión con base en reglas jurídicas. ${ }^{54}$

Del mismo modo, esta postura se diferencia con la de Frank dado que los jueces no deciden con base a factores personales, sino por medio de situaciones similares. ${ }^{55}$ Es decir, no basta con analizar los estímulos que afectan al operador judicial y su personalidad, sino que es necesario considerar cuestiones de carácter general, esto es, posiciones compartidas con otros jueces, los cuales a su vez marcan un patrón sociológico que se asienta en los hechos del caso. En consecuencia, este planteamiento se enmarca en la denominada ala

53 Leiter, Brian, Rethinking legal realism..., cit., p, 278

54 Ibidem, p, 285.

55 Núñez, Álvaro, El realismo jurídico de Brian Leiter, cit., p, 441. 
FILOSOFÍA DEL DERECHO NATURALIZADA...

sociológica del realismo la cual es opuesta al ala idiosincrática defendida por Frank. ${ }^{56}$

Otra diferencia enunciada por Leiter respecto a la distorsión que se ha generado sobre el mismo tiene que ver con la indeterminación del derecho, ya que, para los realistas no existe una indeterminación radical del derecho - la que a su vez trae consigo un menor grado de seguridad jurídica- ${ }^{57}$ puesto que existen casos en los cuales no hay discusión sobre cual deba ser la solución, ${ }^{58}$ dicho, en otros términos, se sabe con certeza qué decisión tomará el operador judicial.

Así las cosas, la indeterminación judicial parte de la clase de razones jurídicas a saber: $i$ ) fuentes legítimas del derecho, ii) métodos de interpretación de fuentes, iii) métodos de apreciación e interpretación de los hechos, iv) métodos de razonamiento deductivo. ${ }^{59} \mathrm{Te}$ niendo en cuenta este tipo de clases, la indeterminación se presenta de dos formas: i) racional y ii) causal. En tal sentido, el derecho es indeterminado racionalmente, si la clase es insuficiente para explicar una única decisión, esto se debe principalmente a los métodos legítimos de interpretación, aunque también se puede deber a un problema en la identificación de la fuente aplicable. ${ }^{60}$ Un ejemplo clásico que sirve para entender esto es el acuñado en el debate Hart $v s$. Fuller, ${ }^{61}$ según el cual existe una norma jurídica que prohíbe la entrada de los vehículos en los parques, en tal sentido, en este caso es posible llegar a dos decisiones distintas ya que por ejemplo si un juez aplica un canon de interpretación literal están prohibidos en el

56 Leiter, Brian, Realismo jurídico estadounidense, cit., p, 249.

57 Sobre la idea gradual de seguridad jurídica véase, Gometz, Gianmarco, La certeza jurídica como previsibilidad, trad. de Diego Moreno Cruz y Diego Dei Vecchi, Madrid, Marcial Pons, 2012, p. 271.

58 Núñez, Álvaro, “¿Deciden los jueces por razones políticas?”, Jurídicas, Manizales, vol. 9, núm. 2, julio-diciembre de 2012, pp. 13-31.

59 Leiter, Brian, “Legal Indeterminacy”, Legal theory, Cambridge, vol. 1, núm. 4, diciembre de 1995, pp. 481-492.

60 Leiter, Brian, Naturalismo y teoría del derecho, trad. de Giovanni Battista Ratti, Madrid, Marcial Pons, 2012, pp. 42 y 43.

61 Hart, Herbert y Fuller, Lon, El debate Hart-Fuller, trad. de Jorge González Jácome, Bogotá, Universidad Externado de Colombia, 2016, p. 166. 
parque todos los artefactos que se subsuman en la categoría vehículo sin importar ningún tipo de razón excluyente, en cambio si el operador jurídico acoge un canon teleológico, puede permitir la entrada de ciertos vehículos que cumplan con la finalidad de la norma a saber, proteger la vida de las personas que transiten en el parque, en tal sentido para este operador estaría permitido que ingrese al parque una ambulancia la cual entra para ayudar a una persona que sufrió un accidente.

Por su parte, la indeterminación causal se presenta cuando, la clase no explica de forma causal la decisión del juez, ${ }^{62}$ al respecto téngase en cuenta el siguiente ejemplo: en el colegio $\mathrm{X}$ de la ciudad de Bogotá, Colombia se le ordena al estudiante Y que se corte el cabello debido a que el manual de convivencia establece que el corte de los caballeros debe ser corto, el joven Y estima que tal decisión es inconstitucional porque atenta contra el derecho fundamental al libre desarrollo de la personalidad, por lo que impone una acción de tutela para que le ampare su derecho fundamental. El juez de este caso tiene por lo menos las siguientes posibilidades:

i) No tutelar el derecho debido a que el derecho fundamental al libre desarrollo de la personalidad no es absoluto, y no se está violando en este caso debido a que, el colegio tiene el deber de formar integralmente a los menores y esto implica exigirles un corte de cabello corto.

ii) Tutelar el derecho, debido a que efectivamente el manual de convivencia si vulnera ese derecho fundamental, y por lo tanto permitirle a Y tener el cabello largo.

iii) Tutelar el derecho, pero con ciertas limitantes, como exigirle al estudiante Y que se recoja el cabello durante la jornada escolar.

En este punto, es donde cobran relevancia las posturas de Frank, dado que gracias a esta indeterminación causal la personalidad del juez y la forma en la que este aprecie la pruebas traerán consigo que la decisión no sea la misma si fallan dos operadores judiciales distintos, así pues, tomando el ejemplo anterior si este caso imaginario 
le corresponde a un juez conservador seguramente no tutele el derecho de acuerdo con los argumentos expresados en la posibilidad i), en cambio, si le corresponde a un juez de corte liberal, lo más probable es que este tutele el derecho con fundamento al principio de autonomía el cual hace parte esencial del derecho fundamental al libre desarrollo de la personalidad.

En este estado de la discusión, y con base a la reconstrucción hecha por Leiter, el realismo jurídico como teoría del derecho, está marcado por dos corrientes filosóficas, por un lado se encuentra el naturalismo, que propende porque dentro de la teoría realista haya elementos de investigación empírica propios de las ciencias naturales y sociales, mientras que por el otro, para el pragmatismo lo esencial de la teoría realista es construir una teoría que permita predecir como las cortes van a decidir. ${ }^{63}$ Leiter se encuadra en la corriente naturalista a consecuencia de que con, la aplicación de dicho método se puede obtener un conocimiento genuino y por lo tanto todas las demás disciplinas deberías emplear el mismo método. ${ }^{64}$ No obstante naturalismo y pragmatismo no son visiones distintas sino complementarias en la medida en que la aplicación del método naturalista ayuda a la predicción de las decisiones judiciales. ${ }^{65}$

Así las cosas, la filosofía del derecho naturalizada propuesta por Leiter se caracteriza por:

i) Propender que la filosofía del derecho se acoja al giro naturalista de la filosofía y por lo tanto abandone el análisis conceptual predominante en la misma, ${ }^{66}$ debido a que éste - entendido como una herramienta que suministra definiciones de diversos conceptos, empleados por el derecho con la finalidad de dar una clarificación acerca de la naturaleza del derecho- ${ }^{67}$

63 Leiter, Brian, Rethinking legal realism ..., cit., p. 286.

64 Leiter, Brian, Realismo jurídico estadounidense, cit., p, 242.

65 Ratti, Giovanni, op. cit., 14.

66 López, Nicolás, Naturalismo y derecho..., cit., p, 22.

67 Himma, Kenneth, "Conceptual Jurisprudence. An Introduction to Conceptual Analysis and Methodology in Legal Theory" Revus. Journal for Constitutional Theory and Philosophy of Law, Liubliana, núm. 26, 2015, p. 65. 
no garantiza un mayor conocimiento sobre los asuntos que se aborden. ${ }^{68}$

ii) La finalidad de lo anterior radica en el hecho de imponer al derecho las demarcaciones epistemológicas propias de las ciencias naturales, con el propósito de darle una mayor cientificidad al mismo. ${ }^{69}$ De modo similar, este interés también fue compartido por los realistas jurídicos escandinavos, debido a que Ross estimaba que el método científico ayuda a conocer los hechos del mundo, es decir la ciencia jurídica es una ciencia social de hechos. ${ }^{70}$

iii) Al conjugar el punto i y ii, se extrae que la propuesta naturalista en el campo de la teoría del derecho, naturalizada reside en el hecho de que ésta permite un mayor estándar de validación y justificación epistémica, debido a su aplicación de la denominada nueva ciencia cognitiva. ${ }^{71}$

iv) Esta visión no implica un cambio en el objeto de estudio de la teoría jurídica, sino más bien arroja una nueva forma de analizar los problemas propios de la disciplina ${ }^{72}$

v) Centrar su atención en la predicción de las decisiones judiciales, que se puede realizar de dos formas: 1) describiendo como los jueces deciden sus decisiones y hasta qué punto era previsible tal decisión, y 2) organizando una metodología de carácter prescriptivo que permita predecir como los operadores judiciales van a fallar. ${ }^{73}$ Bajo esta óptica y atendiendo a la clasificación realizada por Brian Bix respeto a las formas de hacer teoría jurídica, ${ }^{74}$ la teoría Leiteriana del derecho es una de carácter

${ }^{68}$ Núñez, Álvaro, El realismo jurídico de Brian Leiter, cit., p. 449.

69 López, Nicolás, Naturalismo y derecho..., cit., pp. 22 y 23.

70 Ross, Alf, Hacia una ciencia realista del derecho, critica del dualismo en el derecho, traducción de Julio Barbosa, Buenos Aires, Abeledo Perrot, 1961, p. 333.

71 Cáceres, Enrique y Montemayor, Carlos, op. cit., p. 138.

72 Leiter, Brian, Naturalismo y teoría del derecho, cit., p. 255.

73 Núñez, Álvaro, El realismo jurídico de Brian Leiter, cit., p. 454.

74 Bix, Brian, Teoría del Derecho: tipos y propósitos, trad. de Erica Frontini, Isonomía: Revista de Teoría y Filosofía del Derecho, México, 2006, núm. 25, Octubre, p. 57-68. 
FILOSOFÍA DEL DERECHO NATURALIZADA...

descriptivo y/o prescriptivo que rechaza la teoría conceptual del derecho.

\section{PROBLEMAS Y CRÍTICAS DE LA FILOSOFÍA DEL DERECHO NATURALIZADA}

Una vez analizada la propuesta de una filosofía del derecho naturalizada, corresponde ahora enunciar los principales problemas y críticas a los que se enfrenta. En primer lugar, al implementar los métodos propios de las ciencias naturales en el derecho y elevar los estándares de validación de las teorías jurídicas se ésta ante una reducción de la filosofía jurídica y la teoría del derecho por parte de la sociología jurídica. Esto se debe precisamente a que existen ciertas materias o problemas de la teoría jurídica que si se validan de forma empírica entrarían en el terreno de la sociología jurídica. Sin embargo, vale la pena aclarar que a pesar de ser disciplinas autónomas e independientes entre sí, dado que la teoría jurídica se encarga de estudiar al derecho en sí mismo, y la sociología jurídica estudia al derecho con lo que lo rodea, ${ }^{75}$ es decir con su entorno, estas disciplinas guardan relación, debido a que en determinadas situaciones la sociología jurídica, aporta determinados datos sobre temas en concreto a saber: eficacia de las normas jurídicas, los cuales son tomados por la filosofía del derecho y a partir de estos se desarrollan puntos de vista críticos, los cuales tienen la finalidad de mejorar el proceso de creación y aplicación de las normas jurídicas. ${ }^{76}$

Así pues, la sociología jurídica al tener como campos de estudio los siguientes temas: i) perspectiva histórica de la sociología jurídica, ii) normatividad social y derecho, iii) sociología general y derecho, iv) técnicas de investigación social en el campo jurídico, v) función, eficacia y aplicación del derecho, vi) sociología de la profesión jurídica, ${ }^{77}$ ocasiona que, la propuesta hecha por la filosofía del

75 Soriano, Ramón, Sociología del derecho, Barcelona, Ariel, 1997, p. 31.

76 Prieto, Camilo, “¿Es la teoría de la argumentación jurídica una superación de la teoría del derecho?", Revista Gaceta Jurídica, Bucaramanga, vol. 8, enero-diciembre de 2015, pp. 15-22.

77 Soriano, Ramón, op. cit., pp. 37 y 38. 
derecho naturalizada se enmarca dentro de la misma, puesto que al pretender elevar los estándares de validación empírica de las investigaciones filosóficas, se estaría analizando más que el aporte que arroje determinada investigación, su estándar de validación, es decir si está cumpliendo o no.

Lo antepuesto no debe confundirse con la operatividad de las teorías, puesto que, lo que se busca con la formulación de teorías es que éstas sean tanto operativas como prácticas. ${ }^{78}$ En este sentido, por ejemplo, es muy distinto preguntarse si la teoría de los derechos fundamentales de Robert Alexy, ${ }^{79}$ es operativa y práctica, a si la misma está siendo aplicada de forma rigurosa por el tribunal constitucional de Alemania.

Así las cosas, y centrándose en la reducción de la filosofía jurídica por parte de la sociología del derecho, se encuentra como ejemplo de esta situación, la investigación realizada por Enrique Cáceres y Carlos Montemayor, ${ }^{80}$ en la que aplican y abordan el método de la filosofía del derecho naturalizada, empero tal labor se realiza sobre un problema clásico de la sociología jurídica como lo es, la eficacia de las normas jurídicas. Situación que reafirma la crítica reduccionista. Sin embargo, es preciso aclarar que tal situación, no implica que todas las investigaciones que se realicen sobre la eficacia de las normas jurídicas se circunscriben dentro de la sociología jurídica, ya que puede verse desde otros enfoques: i) el análisis económico del derecho, ${ }^{81}$ ii) el enfoque conceptual, el cual es contrario al enfoque fáctico - propio de la sociología jurídica- y tiene por finalidad analizar la definición y alcance de la eficacia de las normas jurídicas. ${ }^{82}$

Tal reducción se debe a la fundamentación empleada por Leiter, es decir, al tomar como referente principal a Quine se incurre en el

78 Agudelo, Oscar, "Los calificativos del derecho en las formas de investigación jurídica", en Agudelo, Oscar (ed.), La pregunta por el método: Derecho y metodología de la investigación, Bogotá, Universidad Católica de Colombia, 2018, pp. 17-44.

79 Alexy, Robert, Teoría de los derechos fundamentales, 2a. ed., traducción de Carlos Bernal Pulido, Madrid, Centro de estudios políticos y constitucionales, 2008.

80 Cáceres, Enrique y Montemayor, Carlos, op. cit., p, 137-165.

81 Agudelo, Oscar, op. cit., p, 28.

82 Navarro, Pablo, "Normas, sistemas jurídicos y eficacia" Crítica, revista hispanoamericana de filosofía, México, 1990, vol. XXII, núm. 64, abril de 1990, pp. 41-53. 
FILOSOFÍA DEL DERECHO NATURALIZADA...

mismo error que este, a consecuencia de que, en el caso de Quine, se presenta la reducción de la filosofía a alguna ciencia empírica. ${ }^{83}$ Esto se debe a que Quine representa la versión más radical del naturalismo, posición que esta contrapuesta a las versiones más moderadas de naturalización, según las cuales, filosofía y ciencia tienen tanto puntos en común, como puntos donde son distinguibles una de la otra ${ }^{84}$ Así pues, a la hora de construir una filosofía del derecho naturalizada, es necesario tomar en cuenta las versiones moderadas de la naturalización filosófica, tal como lo hace Daniel González Lagier, ${ }^{85}$ quien, por medio de la aplicación de los puntos de intersección entre derecho y neurociencia, pretende naturalizar tanto la filosofía practica como la responsabilidad, ${ }^{86}$ para luego aplicar tales posturales a campos en específico de las ciencias jurídicas como lo es el derecho penal. ${ }^{87}$

En síntesis una versión moderada de naturalización filosófica propenderá por, no darle en ningún momento la espalda a la ciencia, puesto que es necesario reconocer cierta influencia a los descubrimientos empíricos que se puedan generar en el campo de la filosofía, es decir, no se debe contradecir lo que ya se ha comprobado empíricamente, ${ }^{88}$ y comprender que hay zonas que le competen a la filosofía y donde no es posible que la ciencia llegue, puesto que los

83 González, Daniel, "Sobre los presupuestos filosóficos de las pruebas neurocientíficas (A propósito de «Proceso y neurociencia. Aspectos generales», de M. Taruffo)", en Ferrer, Jordi y Vázquez, Carmen (ed.), Debatiendo con Taruffo, Madrid, Marcial Pons, 2016, pp. 385-412.

84 Ibidem, p. 385.

85 Daniel González Lagier encuentra tres restos que debe afrontar la naturalización de la responsabilidad y son: “¿Puede una filosofía práctica naturalizada dar cuenta de la normatividad? ¿Qué respuesta da la neurociencia al problema del libre albedrio? ¿Qué aporta la neurociencia a la prueba de los estados mentales necesarios para la atribución de responsabilidad?". González, Daniel, "Sobre los presupuestos filosóficos de las pruebas neurocientíficas..., cit., p, 387.

86 Idem.

87 González, Daniel, “Tres retos de la neurociencia para el Derecho penal”, Anuario de filosofía del derecho, Valencia, 2018, núm. XXXIV, pp. 43-72.

88 González, Daniel, Sobre los presupuestos filosóficos de las pruebas neurocientíficas..., cit., p, 407. 


\section{CAMILO HUMBERTO PRIETO FETIVA}

avances de la ciencia deben ser interpretados por la filosofía, y es ahí donde la filosofía realiza una tarea de control. ${ }^{89}$

Otro reparo, que se relaciona bastante con el anterior, reside en el hecho de que no todos los problemas de la teoría jurídica y la filosofía del derecho son abordables desde esta óptica, en la medida de que éstos no requieres una elevación en los estándares de validación — de ahí que se emplee el método de análisis conceptual como lo han hecho Hart o Raz-, tal es el caso de la validez de las normas jurídicas, la justificación de las decisiones judiciales, las fórmulas de ponderación de derechos fundamentales, la interpretación jurídica, la dogmática jurídica, la teorías de la justicia entre otros. Cosa distinta es el impacto que estas generan en la sociedad, debido a que no es lo mismo preguntarse ¿Qué métodos de justificación de decisiones judiciales existen? 0 ¿Qué se entiende por validez de las normas jurídicas? A interrogarse ¿Cuál es el impacto que genera en la ciudad de Bogotá los métodos de justificación de decisiones judiciales utilizados por los jueces civiles municipales? 0 ¿si es eficaz el código civil colombiano? Esto se debe, a que más que desarrollar una filosofía del derecho naturalizada Leiter desarrolla una naturalización del proceso de adjudicación, que se centra en el tema de la predictibilidad, y no en otros aspectos concernientes a la decisión judicial, como lo relativo a la justificación de la decisión que toma el operador judicial.

La tercera crítica se centra más en la propuesta de Leiter respecto a lo ateniente a la predictibilidad de las decisiones judiciales, ${ }^{90}$ de-

89 Vázquez, Rodolfo, "Relatoría del tema uno, «¿Qué filosofía del Derecho para el Mundo Latino?», Revista i-Latina, Alicante, 2017, núm. 0, pp. 1-22.

90 Debe precisarse que el método sociológico de predicción de Leiter es sólo uno de los enfoques existentes para predecir y explicar decisiones judiciales, los otros dos enfoques son: el psicológico y el económico, el enfoque psicológico es heredero de los trabajos de Jerome Frank y parte de la idea de predecir decisiones a partir de las herramientas psicocognitivas como las denominadas heurísticas o atajos mentales que emplean los operadores judiciales al momento de fallar, en cambio el enfoque económico si bien es cierto no es muy útil para predecir decisiones judiciales si lo es para explicarlas, puesto que toma como punto de partida la utilidad esperada por el operador judicial y lo sitúa como un agente racional. Al respecto véase Moreno, Diego, "Three Realistic Strategies for Explaining and Predicting Judicial Decisions", en Mathis, Klaus (ed.), Law and Economics in Europe. Foundations 
bido a que ésta es bastante compleja y muy poco útil debido al carácter temporal de los jueces, y en dados casos a la existencia de contradicciones ideológicas en los mismos, puesto que en determinadas ocasiones jueces liberales fallan como conservadores y viceversa. Esto refleja un problema con la operatividad de la teoría. Igualmente, Leiter, en ningún momento postula los elementos para tener en cuenta para predecir una decisión judicial, únicamente resalta el hecho de que los jueces deciden con base a casos similares, por ello si se quiere predecir una decisión de la Corte Constitucional Colombiana, en primer lugar, es necesario determinar que variables de medición tienen los 9 magistrados de la corte constitucional, es decir, ¿Cuál es su edad? ¿Cuál es su nivel de educación? ¿De qué universidad son egresados? ¿Si son casados o solteros? ¿Qué inclinaciones sexuales tienen? ¿Cuál es su ideología política? ¿Qué tendencias religiosas poseen? ¿Cuál es el precedente existente dentro del caso que se pretende resolver? Entre otras. En segundo lugar, se necesita establecer en qué medida estos patrones se relacionan con la materia de discusión del caso, y finalmente al unir los datos y consideraciones realizadas, se podría predecir eventualmente la decisión de cada magistrado.

Sin embargo, estas consideraciones no aseguran que la predictibilidad de la decisión sea acertada, dado que no existe un método o herramienta que permita establecer sin errores cómo las personas actúan.

\section{CONCLUSIONES}

La filosofía del derecho naturalizada formulada por Brian Leiter, al ser una heredera - mas no una reiteración- de los postulados del realismo jurídico norteamericano, consiste en un intento de dar el giro naturalista en las ciencias jurídicas debido a la ausencia de este, y de elevar los estándares de validación de las investigaciones que se realicen, lo que acarrea una mayor cientificidad del derecho. No obstante, tal situación reduce la filosofía del

and Applications, Dordrecht, Springer, 2014, pp. 93-124. 
derecho y a la teoría jurídica a una sociología jurídica, puesto que Leiter incurre en el mismo error de Quine a saber, reducir a la filosofía a una ciencia empírica. Por ello si lo que se quiere dar es un giro hacia el naturalismo, es necesario acudir a posturas moderadas de naturalización — tal como lo hace Daniel González Lagier-, las cuales no acarrean el problema del reduccionismo, puesto que son conscientes de que filosofía y ciencia son disciplinas diferentes, que tienen puntos en común, pero que tales puntos en común no implican una preponderancia de una sobre la otra.

Adicionalmente, no todos los problemas de la filosofía y teoría del derecho pueden ser abordados desde esta óptica, y las herramientas que ofrece en lo ateniente a la predecibilidad de las decisiones judiciales resultan bastante complejas y poco útiles, lo que muestra su carencia de carácter omnicomprensivo. Empero esto no conduce a un rechazo de esta teoría o a la inoperatividad de esta, sino más bien a una reconsideración, y es que se aplique en casos con determinada relevancia tanto nacional e internacional, teniendo en cuenta las variables mencionadas en el presente trabajo. Además, no sobra enfatizar, que tales variables en ningún momento garantizan una predictibilidad de las decisiones judiciales en un cien por ciento.

A este tenor, existen ciertos puntos que requieren un mayor desarrollo y que como consecuencia de tal situación quedan como nuevos subproblemas a tratar:

El primero de éstos de estos es ¿si la filosofía del derecho naturalizada - propuesta por Brian Leiter - embrolla una re-validación del contexto de descubrimiento dentro del proceso de decisión judicial? ya que esta postura incorpora una especial importancia a factores no jurídicos que determinan la toma de decisiones judiciales, mas no a la justificación de la misma, a consecuencia de que en la gran mayoría de los textos referentes a argumentación jurídica se dedican una gran cantidad de hojas a analizar los métodos de justificación de la decisión judicial y muy pocas a abordar lo referente a la predictibilidad de las decisiones jurídicas.

El segundo subproblema, es ¿hasta qué punto la filosofía del derecho naturalizada también debería abordar problemas propios de la epistemología jurídica? es decir ¿existe una epistemología jurídica naturalizada? más específicamente lo referente a la validación del 
conocimiento obtenido por el operador judicial a partir de los medios de prueba, tarea en la cual sus postulados parecerían tener una mayor aplicabilidad y un posible futuro desarrollo teórico, siempre y cuando se acoja una versión moderada de naturalización.

Y finalmente, el tercer subproblema es ¿si la filosofía del derecho naturalizada Leiteriana, en sí misma es una ciencia o más bien es una técnica que permite en una menor o mayor medida dependiendo del caso predecir decisiones judiciales? Situación que es bastante problemática en la medida en que reaviva el viejo debate acerca si el derecho es o no una ciencia.

\section{REFERENCIAS}

Agudelo, Oscar, "Los calificativos del derecho en las formas de investigación jurídica”, en Agudelo, Oscar (ed.), La pregunta por el método: Derecho y metodología de la investigación, Bogotá, Universidad Católica de Colombia, 2018.

Agudelo, Oscar et al., Teoría jurídica y enseñanza del derecho, Bogotá, Universidad Católica de Colombia, 2015.

Alchourrón, Carlos y Bulygin, Eugenio, Sistemas normativos. Introducción a la metodología de las ciencias jurídicas, 2a. ed., Buenos Aires, Editorial Astrea, 2012.

AlEXY, Robert, Teoría de los derechos fundamentales, 2a. ed., trad. de Carlos Bernal Pulido, Madrid, Centro de estudios políticos y constitucionales, 2008.

BARberis, Mauro, “Un poco del realismo jurídico Genoves”, en Ferrer, Jordi y Ratti, Giovanni (eds.), El realismo jurídico genovés, Madrid, Marcial Pons, 2011.

BIX, Brian, "Algunas reflexiones sobre metodología en Teoría del Derecho", trad. de Pablo Navarro, Doxa. Cuadernos de Filosofía del Derecho, Alicante, 2003, núm. 26.

BIX, Brian, "Teoría del Derecho: tipos y propósitos", trad. de Erica Frontini, Isonomía: revista de teoría y filosofía del derecho, México, 2006, núm. 25, octubre. 
CÁceres, Enrique y Montemayor Carlos, "Pasos hacia una naturalización cognitiva en la filosofía del derecho (indexicalidad consciente e inconsciente: una revisión a la eficacia normativa)", Problema. Anuario de filosofía y teoría del derecho, México, núm. 10, enero-diciembre de 2016.

CARpintero, Francisco, Historia del derecho natural: un ensayo, México, UNAM, 1999.

Dworkin, Ronald, Los derechos en serio, trad. de Marta Gustavino, Barcelona, Ariel, 2014.

Ezquiaga, Francisco, "Los juicios de valor en la decisión judicial", Anuario de Filosofía del Derecho, Valencia, 1984, núm. I.

Ferrajoli, Luigi, Principia iuris. Teoría del derecho y de la democracia, 2a. ed., trad. de Perfecto Andrés Ibáñez, Alfonso Ruiz Miguel et al., Madrid, Trotta, 2016, t, 1. Teoría del derecho.

FINNIS, John, Estudios de teoría del derecho natural, edición de Javier Saldaña y Carlos Massini, México, UNAM, 2017.

Frank, Jerome, "Are judges human? Part two: as through a class darkly", University of Pennsylvania Law Review, Philadelphia, vol. 80, núm. 2, diciembre de 1931.

FrANK, Jerome, Law \& the modern mind, New Brunswick, Transaction Publishers, 2009.

GrAY, J, The nature and sources of the law, New York, The Columbia University press, 1909.

Gometz, Gianmarco, La certeza jurídica como previsibilidad, trad. de Diego Moreno Cruz y Diego Dei Vecchi, Madrid, Marcial Pons, 2012.

GonzÁLEz, Daniel, "Filosofía y (Neuro)ciencia: Sobre la "naturalización" de la filosofía práctica", ponencia presentada en el primer congreso de filosofía del derecho para el mundo latino, Alicante, 2016. Disponible en: http://iusfilosofiamundolatino.ua.es/download/Filosof\%C3\%ADa\%20y\%20(Neuro) ciencia.\%20Sobre\%20la\%2 0naturalizaci\%C3\%B3n\%20 de\%20la\%20filosof\%C3\%ADa\%20pr\%C3\%A1ctica.pdf. 
GonZÁlEZ, Daniel, “Sobre los presupuestos filosóficos de las pruebas neurocientíficas (A propósito de «Proceso y neurociencia. Aspectos generales», de M. Taruffo)", en FERRER, Jordi y VÁzQUEZ, Carmen (ed.), Debatiendo con Taruffo, Madrid, Marcial Pons, 2016.

GonzÁLEZ, Daniel, “Tres retos de la neurociencia para el Derecho penal", Anuario de Filosofía del Derecho, Valencia, 2018, núm. XXXIV.

GuASTINI, Riccardo, Distinguiendo: estudios de teoría y metateoría del derecho, trad. de Jordi Ferrer Beltrán, Barcelona, Gedisa, 1999.

GuASTInI, Riccardo, La sintaxis del derecho, trad. de Álvaro Núñez, Madrid, Marcial Pons, 2016.

HART, Herbert, El concepto de derecho, 3a. ed., trad. de Genaro Carríó, Buenos Aires, Abeledo Perrot, 2009.

HART, Herbert y Fuller, Lon, El debate Hart-Fuller, trad. de Jorge González Jácome, Bogotá, Universidad Externado de Colombia, 2016.

HaWking, Stephen y Mlodinow, Leonard, Brevísima historia del tiempo, trad. de David Jou, Barcelona, Critica, 2015.

Holmes, Oliver, La senda del derecho, trad. de José Ignacio Solar Cayón, Madrid, Marcial Pons, 2012.

HimmA, Kenneth, "Conceptual Jurisprudence. An Introduction to Conceptual Analysis and Met-hodology in Legal Theory", Revus. Journal for Constitutional Theory and Philosophy of Law, Liubliana, núm. 26, 2015.

Kunn, Thomas, La estructura de las revoluciones científicas, trad. de Agustín Contin, Buenos Aires, Fondo de Cultura Económica, 2004.

LAVILlA, Juan, "Sobre el "case-method" para la enseñanza del Derecho. La experiencia de la "Har-vard Law School"”, Revista de Administración Pública, Madrid, núm. 117, septiembre-diciembre de 1988. 
LEITER Brian, "Legal Indeterminacy”, Legal Theory, Cambridge, vol. 1, núm. 4, diciembre de 1995.

LEITER Brian, Naturalismo y teoría del derecho, trad. de Giovanni Battista Ratti, Madrid, Marcial Pons, 2012.

LEITER Brian, "Objectivity, morality, and adjudication", en Leiter Brian (ed.), Objectivity in law and morals, Cambridge, Cambridge University Press, 2007.

LEITER Brian, "Realismo jurídico estadounidense", trad. de Jorge Fabra, en Fabra, Jorge y Núñez, Álvaro (eds.), Enciclopedia de filosofía y teoría del derecho, México, UNAM, 2015, vol. 1.

LEITER Brian, "Rethinking legal realism: toward a naturalized jurisprudence", Texas Law Review, Austin, vol. 76, num. 2, diciembre de 1997.

LeITER Brian, "The Naturalistic Turn in Legal Philosophy", Apa Newsletter on Philosophy and Law, Newark, 2001, vol. 00, num. 2, Spring.

LóPEZ, Nicolás, "El naturalismo jurídico como teoría del derecho: red filosófica y conceptual de un cientificismo revisitado", Derecho y humanidades, Santiago, núm. 22, julio-diciembre de 2013.

LóPEZ, Nicolás, "Naturalismo y derecho: una perspectiva científica en la teoría del derecho norteamericano del siglo XXI", Revista de Ciencias Sociales, Valparaíso, 2016, núm. 68.

LóPEZ, José, "El formalismo en la teoría jurídica estadounidense" Anuario de filosofía del derecho, Valencia, 2001, núm. XVIII, pp. 267-300.

MAdDy, Penelope, Second Philosophy: A Naturalistic Method, Oxford, Oxford University Press, 2007.

MAGGE, Bryan, “Las ideas de Quine. Diálogo con W. V. Quine”, en MAGGE, Bryan (ed.), Los hombres detrás de las ideas. Algunos creadores de la filosofía contemporánea, México, Fondo de cultura económica, 1993.

MASSINI, Carlos, La Ley natural y su interpretación contemporánea, Pamplona, EUNSA, 2006. 
MerTz, Elizabeth, "New Legal Realism: Law and Social Science in the New Millennium", en Mertz, Elizabeth et al., The New Legal Realism, Volume I. Translating law-and-society for today's legal practice, Cambridge, Cambridge Universi-ty Press, 2016, pp. 1-25.

Moreno, Diego, "Three Realistic Strategies for Explaining and Predicting Judicial Decisions", en MATHIS, Klaus (ed.), Law and Economics in Europe. Foundations and Applications, Dor-drecht, Springer, 2014, pp. 93-124.

NAVArro, Pablo, "Normas, sistemas jurídicos y eficacia", Crítica, revista hispanoamericana de filosofía, México, vol. XXII, núm. 64, abril de 1990.

NúÑEZ, Álvaro, "Ciencia jurídica realista: modelos y justificación”, Doxa. Cuadernos de filosofía del derecho, Alicante, 2012, núm. 35.

NúÑEZ, Álvaro, “¿Deciden los jueces por razones políticas?”, Jurídicas, Manizales, vol. 9, núm. 2, julio-diciembre de 2012.

NúÑEZ, Álvaro, "El realismo jurídico de Brian Leiter", Diritto e questioni pubbliche, Palermo, 2010, núm. 10.

PRIETo, Camilo, “¿Es la teoría de la argumentación jurídica una superación de la teoría del derecho?", Revista Gaceta Jurídica, Bucaramanga, vol. 8, enero-diciembre de 2015.

Quine, W, "Naturalización de la epistemología”, trad. de Manuel Garrido, en Quine, W, La relatividad ontológica y otros ensayos, Madrid, Tecnos, 2002.

RatTI, Giovanni, "Estudio introductorio", en Leiter, Brian, Naturalismo y teoría del derecho, Madrid, Marcial Pons, 2012.

REDondo, María, "Teorías del derecho e indeterminación normativa", Doxa. Cuadernos de filosofía del derecho, Alicante, 1997, núm. 20.

Ross, Alf, Hacia una ciencia realista del derecho, critica del dualismo en el derecho, trad. de Julio Barbosa, Buenos Aires, Abeledo Perrot, 1961. 
Russell, Bertrand, Análisis filosófico, trad. de Francisco Rodríguez Consuegra, Barcelona, Paidós, 1999.

Soriano, Ramón, Sociología del derecho, Barcelona, Ariel, 1997.

Timm, Ana, "Antiformalismo jurídico, aproximaciones básicas", Revista de derechos fundamentales, Viña del Mar, 2014, núm. 11.

VÁzQuEz, Rodolfo, "Relatoría del tema uno, “QQué filosofía del Derecho para el Mundo Latino?", Revista i-Latina, Alicante, 2017, núm. 0.

VIEHWEG, Theodor, Tópica y jurisprudencia, 2a. ed., traducción de Luis Díez-Picazo, Madrid, Aranzadi, 2007.

Tumonis, Vitalius, "Legal realism \& judicial decision-making”, Jurisprudencija, Wrocławski, 2012, vol. 19, núm. 4.

WELSEN, Hans, Introducción a la filosofía del derecho: derecho natural y justicia material, 2a. ed., trad. de Felipe González Vincén, Madrid, Aguilar, 1979.

Zaremby, Justin, Legal Realism and American Law, Nueva York, Bloomsbury, 2014. 